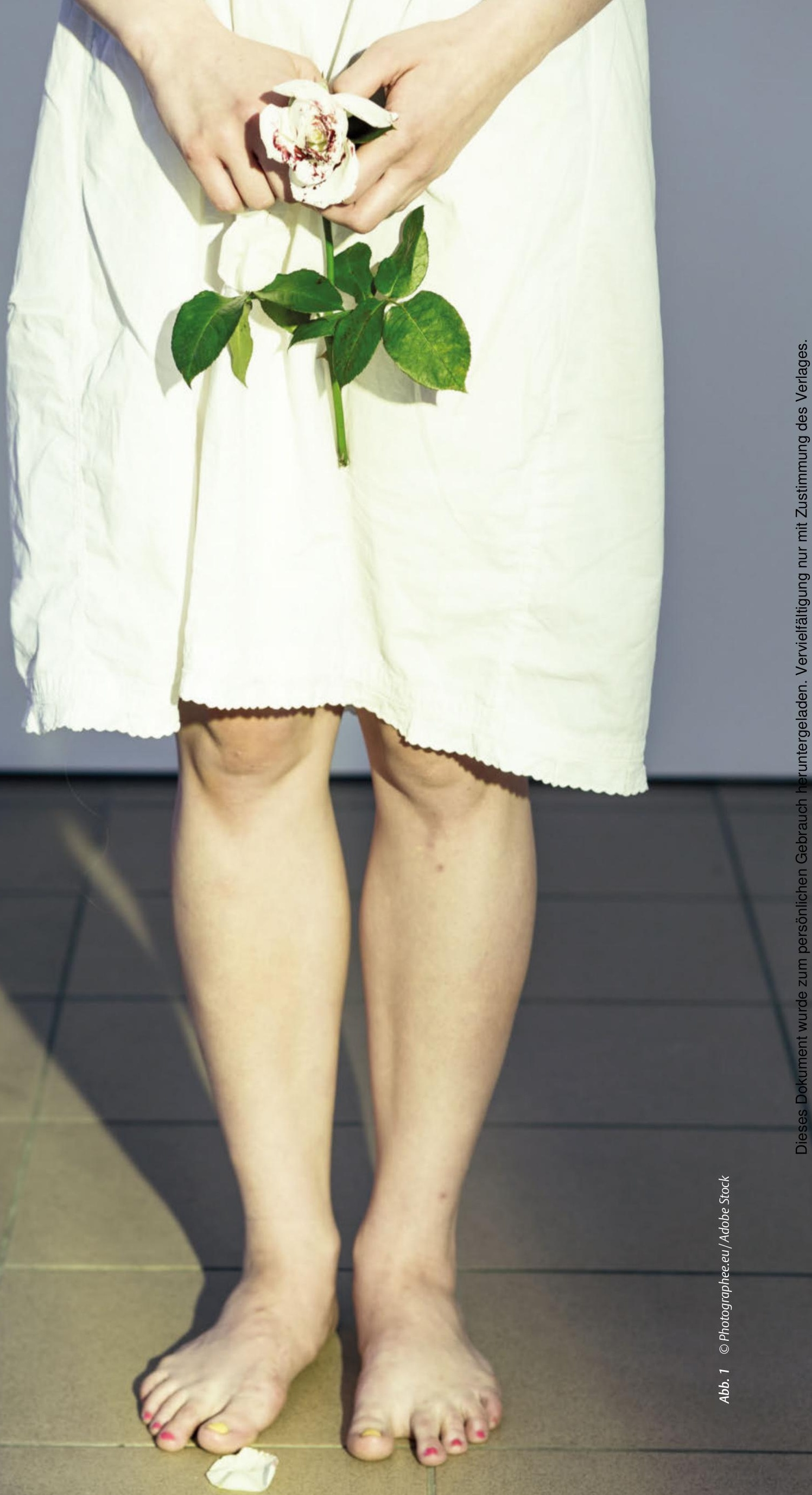




\title{
Multimodale \\ Schmerztherapie bei \\ traumatisierten Patienten
}

\author{
Die emotionale Stabilisierung ist der erste Schritt bei der Behandlung von Traumapatienten - \\ Dann kann im zweiten Schritt aktiv und konfrontativ das Trauma psychotherapeutisch bear- \\ beitet werden \\ Wolfgang Söllner, Sandra Venkat
}

\section{Einleitung}

Bei chronischen Schmerzpatienten wurde in vielen Studien ein hohes Ausmaß verschiedener Formen psychischer Traumatisierung in der Vorgeschichte der Patienten festgestellt [25]. Dies trifft vor allem auf Patienten mit somatoformen Schmerzsyndromen zu [8, 11].

Multimodale Schmerztherapieprogramme (MMST), die edukative, physiotherapeutische, ergotherapeutische und psychotherapeutische Behandlungselemente integrieren [1], haben sich in der Behandlung chronischer „benigner“ Schmerzen als wirksamer erwiesen als rein somatisch orientierte Schmerztherapien oder Behandlungsansätze, in denen nur eine dieser Behand-

\section{Zusammenfassung}

In Schmerzzentren werden häufig Patienten mit Traumatisierung in Kindheit und Jugend und teilweise auch anhaltenden posttraumatischen Belastungsstörungen behandelt. Die Traumatisierung wird oft erst im Rahmen einer multimodalen Schmerztherapie (MMST) erkannt. Die Mitarbeiter von Schmerzeinrichtungen, die eine MMST anbieten, sollten über den Umgang und die Behandlung von traumatisierten Schmerzpatienten geschult werden. Ein zweizeitiges Behandlungsverfahren wird vorgeschlagen: Nach einer MMST sollte eine weiterführende traumatherapeutisch fokussierte psychosomatische Behandlung angeschlossen werden. lungsmethoden angewandt wurde [10, 14]. Aber nur multimodale Programme mit höherer Intensität zeigen diese Effektivität [10]. Die MMST wurde deshalb in die Deutsche Praxisleitlinie zur Behandlung chronischer Rückenschmerzen als Methode der Wahl aufgenommen, wenn „monomodale“ Behandlungen nicht (mehr) wirksam sind [5]. Die multimodale Schmerztherapie hat in den USA, Skandinavien und Deutschland in den letzten 15 Jahren eine rasante Entwicklung genommen. Hunderte von Publikationen sind dazu veröffentlicht worden. Es ist jedoch erstaunlich, wie wenig das Thema der psychischen Traumatisierung in diese Studien und Publikationen Eingang gefunden hat.

In Deutschland wird die Multimodale Schmerztherapie (MMST) meist stationär oder teilstationär angeboten [20] und in geringerem Umfang auch als Kooperationsprojekt in ambulanten Einrichtungen [15]. Die Qualität dieser Programme variiert beträchtlich, insbesondere seit die MMST als Komplexbehandlung zu höheren Erlösen führt und deshalb häufig kostengünstige Programme mit geringer Behandlungsintensität angeboten werden. Eine Ad-hocKommission der Deutschen Schmerzgesellschaft hat deshalb in einem Konsensusverfahren Standards für die MMST entwickelt und publiziert [2]. Intensive Behandlungsprogramme dauern 4-5 Wochen und beinhalten häufig nach 3-6 Monaten ein ein- bis fünftägiges Boosterprogramm zur Festigung der erreichten Veränderungen. Sie sind in- terdisziplinär und enthalten in jedem Fall physio-/ sporttherapeutische, ergotherapeutische, edukative und psychotherapeutische Behandlungselemente in Gruppenund Einzelsitzungen.

Die einzelnen Teile des Behandlungsprogramms müssen eng aufeinander abgestimmt sein. Das aus mehreren Berufsgruppen zusammengesetzte Behandlungsteam muss eine gemeinsame „Philosophie“ der Therapie verinnerlicht haben, die neben der Reduktion der Schmerzen und der schmerzbedingten Einschränkungen die Entwicklung einer bio-psycho-sozialen Sichtweise der Schmerzerkrankung, die körperliche und psychosoziale Aktivierung des Patienten, den Abbau von Hilflosigkeit und Rückzug und die Förderung seiner Ressourcen zum Ziel hat.

Die Wirksamkeit und Prognose einer multimodalen Behandlung hängt stärker von sozialen und psychologischen Faktoren ab als von somatischen Faktoren, insbesondere von der Dauer der Schmerzerkrankung, der Dauer des Krankenstandes [3, 18], einem Wunsch nach Berentung bzw. einem laufenden Rentenverfahren [12], niedrigem sozialem Status [18], hohem Disstress und sozialem Rückzug [19], der Abhängigkeit von Schmerzmedikamenten [7] und der Motivation zur Behandlung [4].

In klinisch orientierten Übersichtsarbeiten wird zwar darauf hingewiesen, dass das Vorliegen einer psychischen Traumatisierung in der Vorgeschichte der Patienten und eine eventuelle posttraumatische Belas- 


\section{Studien belegen ein hohes Ausmaß psychischer} Traumatisierungen bei chronischen Schmerzpatienten, v.a. mit somatoformen Schmerzsyndromen.

tungsstörung die Prognose der Behandlung beeinflussen können und in der Diagnostik und Behandlungsplanung berücksichtigt werden müssen [25, 2]. Es liegen aber kaum Berichte über speziell adaptierte Behandlungsstrategien in einem multimodalen Behandlungssetting oder empirische Untersuchungen dazu vor.

\section{Die Interdisziplinäre Schmerz-} tagesklinik am Klinikum Nürnberg Die Schmerztagesklinik umfasst 10 Behandlungsplätze. Vier Kliniken sind daran beteiligt. Ein Kooperationsvorstand, gebildet aus den Chefärzten der beteiligten Kliniken (Anästhesie, Psychosomatische Medizin, Physikalische Medizin und Neurologie) und der Pflegedienstleitung der Psychosomatik, vertritt die Tagesklinik nach außen und bestimmt die strategische Ausrichtung. Eine interdisziplinäre Leitung, gebildet aus je einem Vertreter der Anästhesie, der Physikalischen Medizin und der Psychosomatik, leitet das operative Geschäft. Das Team setzt sich wie folgt zusammen:

\section{- Fachärzte der Kliniken für}

- Anästhesiologie und Intensivmedizin (80\%; mit Zusatzbezeichnungen

Schmerztherapie und Psychotherapie)

- Psychosomatische Medizin \& Psychotherapie (50\%)

- Physikalische und Rehabilitative Medizin (30\%)

- Neurologie (10\%)

- Psychologische Psychotherapeutinnen (70\% und 45\%)

- Physio-/Sporttherapeuten (2×50\%)

- Ergotherapeutinnen $(2 \times 50 \%)$

- Pain Nurse: $70 \%$

- Sprechstundenhilfen: 50 und 25\% Vor Beginn des eigentlichen Behandlungsprogramms findet ein zweitägiges interdisziplinäres diagnostisches Assessment statt (siehe dazu [6]). Wenn dort die Indikation für eine MMST gestellt wird, werden die Patienten in einem 4-Wochen-Programm plus eine Booster-Woche nach 3 Monaten in geschlossenen Gruppen behandelt. Frauen sind überrepräsentiert. Dies hängt auch damit zusammen, dass ein spezielles Behand- lungsprogramm für türkische Frauen angeboten wird, das von geschulten Dolmetscherinnen unterstützt wird $[16,26]$. In einem 3-Jahreszeitraum wurde bei etwas mehr als einem Drittel der Patienten eine somatoforme Schmerzstörung und bei 7\% der Patienten eine Posttraumatische Belastungsstörung (PTSD) diagnostiziert (Tab. 1). Viele Patienten sind jedoch in Kindheit und Jugend traumatisiert, ohne dass aktuell alle Kriterien für eine PTSD erfüllt sind.

Bei somatoformen Schmerzstörungen ist der Anteil der traumatisierten Patienten höher als bei anderen chronischen Schmerzstörungen. Patienten mit somatoformen Schmerzstörungen zeigen zwar ein Jahr nach Beendigung der MMST weiterhin eine Schmerzreduktion, haben aber hinsichtlich ihrer Lebensqualität und der Depressivität ein schlechteres Behandlungsergebnis als die anderen in der Schmerztagesklinik behandelten Schmerzpatienten [24]. Inwieweit dies auf den höheren Anteil traumatisierter Patienten in der Gruppe der somatoformen Schmerzpatienten zurückzuführen sein könnte, ist bislang noch nicht untersucht.

\section{Fallbericht}

Frau M., eine 33-jährige attraktive junge Frau, wird vom Hausarzt in die Schmerztagesklinik des Klinikums Nürnberg eingewiesen. Das der Schmerzbehandlung immer vorausgehende ein- bis zweitägige interdisziplinäre diagnostische Assessment ergibt die Diagnose einer chronischen Schmerzerkrankung im Chronifizierungsstadium III nach Gerbershagen (wobei der Verdacht geäußert wird, dass psychische Faktoren stark zur Verstärkung und Chronifizierung der Schmerzen beitragen), ein sog. „Failed Back Syndrome“ (die Schmerzen haben sich nach mehreren operativen Eingriffen verstärkt) und einer mittelgradigen Depression mit agoraphobischen Beschwerden.

Frau M. leidet seit 7 Jahren an lumbalen Rückenschmerzen mit Ausstrahlung ins linke Bein. In den letzten Monaten hätten sich die als ziehend und brennend beschriebenen Schmerzen auf den gesamten Rücken und den Schulter-Nacken-Bereich ausgeweitet. Auf der 10-teiligen Visuellen Analogskala ( $1=$ keine Schmerzen und 10=unerträgliche Schmerzen) werden die Schmerzen in Ruhe mit 4 und bei körperlicher Belastung mit 8 angegeben. Sie leide unter ausgeprägten Schlafstörungen, wache nachts alle 1-2 Stunden mit Schmerzen auf. Die Stimmung sei seit Monaten gedrückt, und sie hätte sich von Freizeitaktivitäten und sozialen Kontakten weitgehend zurückgezogen. In engen Räumen würde sie Angst befallen, und sie vermeide es, ins Kaufhaus zu gehen und mit dem Aufzug zu fahren. Nach einer Bandscheibenoperation vor 7 Jahren wurden vier weitere chirurgische Eingriffe durchgeführt, die vor einem Jahr zu einer „Versteifungsoperation“ (Spondylodese) der lumbalen Wirbelsäule geführt haben. Die Patientin nimmt seit Jahren starke Schmerzmedikamente, zuletzt neben einem Opioid-Pflaster zusätzlich ziemlich unkontrolliert höhere Dosen nichtretardierter



Tab. 1 
(also süchtig machender) Opioide. Sie hat keine neurologischen Ausfälle, die Schmerzen bessern sich im Liegen, bei Wärme und bei „Rückzug in eine ruhige Ecke“ (meist die Badewanne). Sie verstärken sich bei Bewegung, körperlicher Anstrengung und nasskaltem Wetter. Frau M. ist Friseurin, habe wegen der Schmerzen ihren eigenen Salon aufgeben müssen und sei nun seit drei Jahren arbeitslos. Sie ist vorübergehend berentet. Die Begutachtung zur Verlängerung der Rente stehe in einigen Monaten an. Frau M. berichtet, dass sie seit neun Jahren mit einem Berufskraftfahrer verheiratet sei, einem ruhigen und verlässlichen Partner, der sie zu nichts dränge. Von Sexualität habe sie sich wegen starker Rücken- und Unterleibsschmerzen zurückgezogen. Sie habe zwei 12 und 10 Jahre alte Kinder. Der Sohn würde an ADHS leiden, sei oft aggressiv und ihr Sorgenkind. Die Tochter würde sich sehr an sie anklammern.

Bei der Aufnahme ins Behandlungsprogramm wirkt Frau M. sehr angespannt, verschlossen und misstrauisch. In der Gruppenpsychotherapie kann sie sich zunächst nicht äußern und nimmt kaum Blickkontakt auf. Darauf angesprochen meint sie, dass die Gruppenbehandlung sie stark unter Druck setze. Sie fühle sich wie eingesperrt, „die Emotionen kochen hoch“. Auch abends könne sie sich nur schwer beruhigen und entspannen. Sie schäme sich, dass sie sich nicht äußern könne und in der Ergotherapie und der medizinischen Trainingstherapie so schlechte Leistungen bringe.

In den Einzelgesprächen mit der Psychotherapeutin kann sie langsam Vertrauen fassen und taut bildlich auf. Sie berichtet, dass die Gruppensitzungen bei ihr starke Ängste und Erinnerungen an schmerzhafte Erlebnisse auslösen würden. Sie habe eine schlimme Kindheit gehabt. Der Vater, den sie sehr geliebt habe, habe sich von der Familie getrennt, als sie 2 Jahre alt war. Er habe sich jedoch im Vorschulalter noch um sie gekümmert. Die Mutter sei der Prostitution nachgegangen, hätte sie gehasst und häufig geschlagen. Ab dem 6. Lebensjahr habe sie den Haushalt führen müssen. Für das Lernen sei keine Zeit geblieben, sie hätte auch keine Freundinnen mit nach Hause nehmen dürfen. Im Alter zwischen 8 und 15 Jahren sei sie von zwei „Freunden“ der Mutter über Jahre sexuell missbraucht worden. Sie habe sich schließlich mit 15 Jahren einem anderen Freund der Mutter mitgeteilt und sei allein zu einer Jugendberatungsstelle gegangen. Der Mutter sei daraufhin das Sorgerecht entzogen worden, und sie sei in ein Heim der Diakonie gekommen.

Die nächsten drei Jahre seien die schönsten ihrer Kindheit gewesen. Mit 18 Jahren sei sie zurück zur Mutter gegangen, die sie gedrängt habe, ,anschaffen zu gehen“. Da sie keine abgeschlossene Ausbildung gehabt hätte (nur Hauptschule ohne Quali), hätte sie als Table-Tänzerin in einem Nachtclub gearbeitet. Dort habe sie ihren späteren Mann kennen gelernt, sei mit ihm zusammengezogen und habe eine Ausbildung als Friseurin gemacht. Sie habe einen eigenen kleinen Salon aufgemacht. Wegen der Schmerzen habe sie diesen aber schließen müssen und müsse heute noch einen hohen Schuldenberg abbezahlen.

Die Besprechung im Therapeutenteam führt dazu, dass die Behandlung von Frau M. modifiziert wird. Ihr wird erlaubt, die Gruppe zu verlassen, wenn es ihr zu viel wird. Es wird mit ihr vereinbart, dass sie in den Gruppensitzungen nicht über die traumatischen Erlebnisse spricht. Alle Behandler fokussieren die Behandlung stark auf ein ressourcenorientiertes und den Selbstwert stärkendes Vorgehen. Ihre Tendenz, sich unter Leistungsdruck zu setzen und sich selbst zu entwerten, solle konsequent thematisiert werden. In der Einzeltherapie fokussiert die Psychotherapeutin auf die emotionale Stabilisierung und Selbstwertstärkung. Den Einsatz von Metaphern, „externalisierenden Interventionen“ und imaginativen Techniken in Anlehnung an Reddemann („Sicherer Ort“, „Tresorübung“) erlebt die Patientin dabei als gewinnbringend und stabilisierend. Aufgrund ihrer vorwiegend auf ihre Defizite fokussierten Wahrnehmung stellt das Aktivieren von früher verfügbaren und heute verschütteten Ressourcen (u.a. Rituale, Tanzen, Gestalten) sowie das Bestärken erfolgversprechender konstruktiver emotionsregulierender Strategien (u.a. gelenkter Selbstdialog, Atemübungen) einen weiteren wichtigen Schwerpunkt dar. An Beispielen von ihr bekannten potenziellen Stresssituationen kann sie bezüglich destabilisierender automatisierter Kognitionen (u.a. „Ich bin schuld.“, „Ich bin damit allein.“, „Ich bin ausgeliefert.“) sensibilisiert werden und diesen mit konstruktiven Strategien (u.a. durch bildhaft dargestelltes „Ausmisten von gedanklichem Müll“ bzw. Veränderung der Einstellung) entgegenwirken. Be- achtlich ist, wie es der Patientin im Umgang mit Intrusionen im geschützten Rahmen zunehmend besser gelingt, rechtzeitig emotionsregulierende Strategien einzusetzen und dabei spürbare Selbstwirksamkeit zu erfahren. Frau M. kann in diesen Gesprächen auch positive Erinnerungen wachrufen, z.B. an eine Tante, die sich um sie gekümmert habe und ihr Geschenke gebracht habe, als sie ein Kind war.

Das Erleben von Rückhalt und Fürsorglichkeit in der Gruppe ermöglicht es der Patientin, neue positive zwischenmenschliche Erfahrungen zu machen, die sie sehr berühren. Frau M. kann sich daraufhin stärker und mit weniger Angst in die Gruppe einbringen. Insbesondere die Bewegungsübungen und die kreative Gestaltungstherapie beginnen ihr Spaß zu machen. Die Bewegungstherapie führt zu einer Veränderung von Bewegungsmustern, Stabilisierung des Rumpfes und einer positiveren Körperwahrnehmung. In der Schmerzedukation hört sie interessiert zu, stellt Fragen und begreift, dass es bei den Schmerzen nicht nur „schwarz oder weiß“ (somatisch oder psychisch) gibt. Ihr zu Beginn ausschließlich somatisch orientiertes Krankheitsmodell (,Wenn ich die richtige Operation bekommen hätte, hätte ich keine Schmerzen“) weicht langsam der Überzeugung, dass auch ihre seelische und soziale Situation die Entwicklung der Schmerzen beeinflussen.

Erfolge im Erlernen und Erleben spannungsregulierender Strategien (Muskelentspannung nach Jacobson) und die über Genuss und Kreativität umsetzbare Selbstfürsorge tragen dazu bei, das Gefühl von Selbstkontrolle und Selbstwirksamkeit zu stärken. Dies führt zu einer Verringerung von Hilflosigkeit und Katastrophisieren und erhöht die Schmerztoleranz.

Die unkontrolliert eingenommenen nichtretardierten Opioide können unter zusätzlicher Gabe eines abendlich eingenommenen sedierenden Antidepressivums bis zum Ende der 4-wöchigen Behandlung ausgeschlichen werden. Am Ende der Behandlung gibt Frau M. zwar nur eine geringe Verminderung der Schmerzstärke, aber eine deutliche Verminderung der schmerzbedingten Einschränkungen und der depressiven Stimmung und einen gebesserten Schlaf an. Die erreichten Verbesserungen halten bis zur Boosterwoche nach 3 Monaten an. Frau M. kann dies als ihren persönlichen Erfolg erleben, fasst wieder mehr Mut und 


\section{Das Erleben von Rückhalt und Fürsorglichkeit in} der Gruppe ermöglichen der Patientin neue positive zwischenmenschliche Erfahrungen zu machen.

entschließt sich, nach einer Wartezeit von weiteren zwei Monaten, die angebotene weiterführende stationäre traumafokussierte psychosomatische Behandlung im Klinikum anzunehmen.

\section{Kommentar zum Fallbericht}

Frau M. hat in ihrer Kindheit und Jugend eine schwere Traumatisierung mit weitreichenden psychischen und sozialen Folgen durchgemacht. Die Aufnahme in die Schmerztagesklinik mit einem intensiven Behandlungsprogramm in einer kleinen geschlossenen Gruppe von 10 Patienten und insbesondere die Gruppenpsychotherapie, in der von manchen Patienten sehr belastende psychische Probleme und Verletzungen mitgeteilt werden, reaktivieren die bisher mehr oder weniger gut verdrängten $\mathrm{Ge}$ fühle von Trauer, Wut, Scham und Schuld. Im Schmerz waren diese Emotionen ,eingekapselt" und zumindest eine Zeit lang einigermaßen „gut aufgehoben“. Nijenhuis [21] bezeichnet diesen Prozess als „somatoforme Dissoziation“. Frau M. konnte sich gleichzeitig als Opfer bestätigt fühlen und ihre Schuldgefühle „besänftigen“. Dieser Abwehrmechanismus der Somatisierung reichte aber nicht aus, sodass Frau M. zunehmend hohe Dosen von Opioiden benötigte, um ihre Übererregbarkeit und ihre Intrusionen („die Emotionen kochen hoch“) in den Griff zu bekommen. Diese Wirkung von Opioiden haben wir in der Behandlung traumatisierter Schmerzpatienten häufig beobachten können, und sie sind sicher mit ein Grund dafür, dass der Entzug von Opiaten bei diesen Patienten so schwierig ist. Das Dilemma ist, dass die Opiate nach anfänglicher Schmerzreduktion nicht selten zu einer erhöhten Schmerzempfindlichkeit und Ausbreitung der Schmerzen führen (Opiatinduzierte Hyperalgesie [27]). Dies kann auch bei Frau M. vermutet werden, weshalb die Reduktion der Opiate ein wesentliches Therapieziel der Behandlung in der Schmerztagesklinik war.

Frau M. verfügte jedoch auch über Ressourcen, die dazu führten, dass sie dann, wenn sie eine vertrauensvolle Beziehung erlebte, zu Kreativität und guten Leistungen fähig war. Die Beziehung zu ihrem Mann und ihren Kindern („sie sind das Wichtigste für mich“) lassen sie Mut und Kraft schöpfen, eine Ausbildung zu beginnen und erfolgreich abzuschließen und einen eigenen Friseursalon zu eröffnen. Es ist nicht selten, dass eine chronische Schmerzkrankheit bei traumatisierten Patienten gerade dann beginnt, wenn sich das Leben zum Besseren wendet.

George Engel hat dies in seiner vielfach zitierten wegweisenden Arbeit zu psychodynamischen Aspekten bei somatoformen Schmerzstörungen als „Pain prone disorder“ [9] bezeichnet und damit die Somatisierung als Folge weitgehend unbewusster Abwehrmechanismen beschrieben, wobei der Schmerz als unbewusste Sühne für Schuldgefühle fungiert und die Tendenz fortsetzt, ein Leben lang im Opferstatus gefangen zu bleiben. Gleichzeitig dient der Schmerz dazu, Zuwendung zu erhalten und als „narzisstische Plombe“ für vermisste oder verlorengegangene sichere Beziehungserfahrungen („gute innere Objekte“ [13]).

Der Aufbau sicherer Beziehungen und der Kampf darum, diese sichere Beziehungserfahrung zu „halten“ (,holding function“ nach [28]) ist deshalb der Kern jeder Traumatherapie [23]. Die unbewusste zur Selbstbestrafung neigende Tendenz vieler komplex traumatisierter Patienten drückt sich häufig in Rückzug, der Tendenz zur Spaltung in gute und böse Ärzte bzw. Therapeuten und Therapieabbruch aus. Dies kann im Kontext einer Behandlung in einem Team nur vermieden werden, wenn sich das Team gut abspricht und koordiniert und gleichsam als Team ein gutes ,inneres Objekt“ (einen ersehnten schützende Familienersatz) für die Patienten bildet.

Der Behandlungserfolg war bei Frau M. sicher nur ein begrenzter und konnte auch nur ein begrenzter sein. Der Zugang zur Behandlung gelang nur darüber, den Schmerz in den Mittelpunkt der Therapie zu stellen. Etwas anderes hätte die Patientin zu diesem
Zeitpunkt nicht akzeptiert. Das Ziel der Behandlung in einem multimodalen Schmerztherapieprogramm kann nur Reduktion von Hilf- und Hoffnungslosigkeit, die Stärkung der emotionalen Kontrolle und des Selbstvertrauens sein sowie die Arbeit an der Motivation, als notwendigen nächsten Schritt, eine intensive traumazentrierte psychosomatische Behandlung in Angriff zu nehmen. Dazu ist es auch notwendig, die Abhängigkeit von opiathaltigen Schmerzmitteln soweit möglich zu reduzieren. Dies ist bei Frau M. nur teilweise gelungen. Ein nachhaltiger Erfolg der MMST war nicht zuletzt durch das laufende Rentenverfahren beschränkt.

\section{Fallbericht (Fortsetzung)}

Kurz vor der stationären psychosomatischen Behandlung erhält Frau M. einen negativen Bescheid bzgl. ihres Rentenantrags. Dies löst Enttäuschung und eine unbändige Wut bei der Patientin aus. Unbewusst bedeutet die Ablehnung des Rentenantrags für sie, dass ihr eine Kompensation für die erlittenen Verletzungen vorenthalten würde und sie es nicht wert wäre, von der Gesellschaft etwas zu erhalten. Wut und Enttäuschung sind so groß, dass sie auch die Klinik und das Behandlungsteam mit der von ihr als feindlich empfundenen „Gesellschaft“ identifiziert (so wie die Patientin ihre Mutter und deren Umgebung in ihrer Kindheit und Jugend erlebt hatte). Sie erwägt, die Behandlung gleich wieder abzubrechen. Die Bearbeitung dieser „Enttäuschungswut“ ist ein Fokus der psychotherapeutischen Arbeit während der stationären Behandlung. Nur so lässt sich ein Arbeitsbündnis herstellen, das es erlaubt, sich auf die Behandlung einzulassen, Nähe zu den Mitpatienten und Therapeuten zuzulassen und in sichereren Beziehungen etwas mehr emotionalen Halt zu finden. Eine familientherapeutische Sitzung mit dem Ehemann und den beiden Kindern trägt dazu bei, das gegenseitige Verständnis zu fördern und den ADHS-kranken und stark verhaltensauffälligen Sohn aus seiner Isolation und Außenseiterrolle herauszuholen, was die Patientin sehr entlastet. Der Sohn übernahm eine in der Familiengeschichte bekannte Rolle und Funktion; der Bruder der Patientin war auf die schiefe Bahn geraten, hatte eine Jugendstrafe erhalten und starb mit 25 Jahren an einer vermutlich selbst verursachten Gasexplosion.

Der zweite Teil der Behandlung diente der weiteren emotionalen Stabilisierung der 


\section{Multimodale Schmerztherapie Praxis zkm}

Patientin im Kontext einer traumatherapeutisch ausgerichteten Behandlung. Die in der Schmerztagesklinik erreichte Verbesserung konnte stabilisiert und die Depressivität und die agoraphobischen Symptome gebessert werden. Die chronischen Schmerzen bestanden jedoch in vermindertem Ausmaß weiter. Inwieweit die mehrfachen chirurgischen Eingriffe eine anhaltende iatrogene Schädigung nach sich gezogen haben, bleibt offen. Die Behandlung muss eine sichere therapeutische Beziehung mit Schmerztherapeuten und Psychotherapeuten über einen längeren Zeitraum hinweg garantieren, als dies eine tagesklinische oder stationäre Behandlung ermöglichen. Frau M. kann sich nach der psychosomatischen Behandlung auf das Angebot einer ambulanten traumatherapeutisch orientierten Behandlung einlassen.

\section{Schlussfolgerungen}

In Schmerzbehandlungszentren werden viele Patienten mit Traumatisierung in Kindheit und Jugend und z.T. auch einer anhaltenden PTSD (,Komplexe Traumafolgestörung“) behandelt. In Einrichtungen, die multimodale Schmerzprogramme anbieten, werden Traumaerfahrungen und Traumafolgestörungen häufig zum ersten Mal diagnostiziert. Traumatisierte Patienten können im Verlauf der multimodalen Behandlung (insbesondere in der Gruppenpsychotherapie) rasch an die Grenzen ihrer bisher einigermaßen erfolgreichen Bewältigungsstrategien geraten, Übererregung (Hyperarousal) und Intrusionen (Flashbacks, Albträume) entwickeln und insgesamt emotional instabil werden. Die Behandlungsprogramme der Multimodalen Schmerztherapie (MMST) müssen deshalb darauf eingestellt und die Mitarbeiter in diesen Einrichtungen diesbezüglich geschult werden.

Die therapeutische Haltung und Strategie muss darauf ausgerichtet sein, im Rahmen der MMST die Patienten zunächst psychisch zu stabilisieren, sie körperlich und psychisch zu aktivieren (Schonhaltung und sozialen Rückzug zu vermindern), die medikamentöse Schmerztherapie anzupassen (Opiode ggf. zu reduzieren oder auszuschleichen) und die Patienten für einen zweiten traumatherapeutisch ausgerichteten Behandlungsschritt zu motivieren und sie darauf vorzubereiten. Dazu müssen die psychotherapeutischen Mitarbeiter von MMST-Programmen in der Anwendung emotional stabilisierender und ressourcenfördernder Verfahren und Techniken geschult werden. Elemente aus Skills-Trainings [17], der Aufmerksamkeitsumfokussierung [29] oder aus imaginativen Verfahren der Traumatherapie (z. B. „Innerer sicherer Ort“, „Tresortechnik“; „Innere Helfer“ [22]) können gut in die Einzeltherapie integriert werden. Es muss aber auch das gesamte Behandlungsteam eine Fortbildung zu den Grundlagen der psychotherapeutischen Arbeit mit Traumapatienten erhalten, um den Patienten im Team einen „haltenden Raum“ bieten zu können. Eine regelmäßig stattfindende Fallbesprechung und Supervision ist unumgänglich, um die häufig auftretenden interaktionellen Schwierigkeiten bei der Behandlung von traumatisierten Schmerzpatienten zu reflektieren, die manchmal negativen Gegenübertragungsreaktionen im Kontext der Psychodynamik eine Opfer-Täter-Spaltung und Übertragung verstehen und dauerhaft eine haltende therapeutische Beziehung herstellen zu können.

Interessenkonflikt: Die Autoren erklären, dass keine wirtschaftlichen oder persönlichen Verbindungen bestehen.

\section{Online zu finden unter}

http://dx.doi.org/10.1055/s-0044-100057

Der leicht modifizierte Artikel ist zuerst erschienen in: Trauma - Zeitschrift für Psychotraumatologie und ihre Anwendungen 2017; 15 (1): 52-63

(c) Asanger Verlag; mit freundlicher Genehmigung

\footnotetext{
Literatur

1 Arnold B, Brinkschmidt T, Casser HR et al. Multimodale Schmerztherapie - Konzepte und Indikationen. Schmerz 2009; 23: 112-120

$\overline{2}$ Arnold B, Brinkschmidt T, Casser HR et al. Multimodale Schmerztherapie für die Behandlung chronischer Schmerzsyndrome - Ein Konsensuspapier der ad-hoc-Kommission Multimodale Schmerztherapie der Deutschen Schmerzgesellschaft zu den Behandlungsinhalten. Schmerz 2014; 28 (5): 459-472

3 Bendix AF, Bendix T, Haestrup C. Can it be predicted which patients with chronic low back pain should be offered tertiary rehabilitation in a functional restoration program? A search for demographic, socioeconomic, and physical predictors. Spine 1998; 23 (16) 1775-1783
} 
$\overline{4}$ Biller N, Arnstein P, Caudill MA et al. Predicting completion of a cognitive-behavioral pain management program by initial measures of a chronic pain patient' $s$ readiness for change. Clin J Pain 2000; 16 (4): 352-359

5 Bundesärztekammer (BÄK), Kassenärztliche Bundesvereinigung (KBV), Arbeitsgemeinschaft der Wissenschaftlichen Medizinischen Fachgesellschaften (AWMF) (2010). Nationale Versorgungsleitlinie Kreuzschmerz - Langfassung. Version 4.2010, zuletzt verändert: August 2013. http://www.kreuzschmerz.versorgungsleitlinien.de.

$\overline{6}$ Casser HR, Arnold B, Brinkschmidt T et al. Interdisziplinäres Assessment für die multimodale Schmerztherapie: Indikationen und Leistungsumfang. Schmerz 2013; 27 (4): 363-370

$\overline{7}$ Cecchi F, Pasquini G, Paperini A et al. Predictors of response to exercise therapy for chronic low back pain: result of a prospective study with one year follow-up. Eur J Phys Rehabil Med 2014; 50 (2): 143-151

8 Egle UT, Ecker-Egle ML, Nickel R et al. Fibromyalgie als Störung der zentralen Schmerz- und Stressverarbeitung. Psychother Psych Med 2004; 54: 137-147

9 Engel GL. „Psychogenic“ pain and the painprone patient. Am J Med 1959; 26: 899-918

$\overline{10}$ Guzmán J, Esmail R, Karjalainen K et al. Multidisciplinary bio-psycho-social rehabilitation for chronic low back pain. Cochrane Database Syst Rev. 2:CD000963; 2006

$\overline{11}$ Häuser W, Arnold B, Eich W et al. Management of fibromyalgia syndrome-an interdisciplinary evidence-based guideline. GMS German Medical Science 2008; 6, DOC 14

$\overline{12}$ Hildebrandt J, Pfingsten M, Saur P et al. Prediction of success from a multidisciplinary treatment program for chronic low back pain. Spine 1997; 22 (9): 990-1001

$\overline{13}$ Hoffmann SO, Egle UT. Psychodynamische Konzepte bei somatoformen Schmerzzuständen. In: Basler HD, Kröner-Herwig B, Franz C, Rehfisch HP, Hrsg. Psychologische Schmerztherapie. Berlin-Heidelberg: Springer; 2013: 119-136

$\overline{14}$ Kamper SJ, Apeldoorn AT, Chiarotto A et al. Multidisciplinary biopsychosocial rehabilitation for chronic low back pain. Cochrane Database Syst Rev, CD000963; 2014

$\overline{15}$ Kayser H, Thoma R, Mertens E et al. Struktur der ambulanten Schmerztherapie in Deutschland. Ergebnisse einer Umfrage. Schmerz 2008; 22 (4): 424-432

$\overline{16}$ Koller W, Venkat S, Söllner W. Effects of a cultural-sensitive pain management program for Turkish women in a day hospital for pain patients (abstract). J Psychosom Res 2011; 70: 601

$\overline{17}$ Linehan MM. Cognitive-behavioral treatment of borderline personality disorder. New York: Guilford; 1993 $\overline{18}$ Mau M, Merkesdal S, Busche T et al. Prognose der sozialmedizinischen Entwicklung ein Jahr nach teilstationärer oder stationärer Rehabilitation wegen Dorsopathie. Rehabilitation 2002; 41 (2/3): 160-166

$\overline{19}$ Michaelson P, Sjölander P, Johansson H. Factors predicting pain reduction in chronic back and neck pain after multimodal treatment. Clin J Pain 2004; 20 (6): 447-454

$\overline{20}$ Nagel B, Pfingsten M, Brinkschmidt T et al. Struktur- und Prozessqualität multimodaler Schmerztherapie - Ergebnisse einer Befragung von schmerztherapeutischen Einrichtungen. Schmerz 2012; 26 (6): 661-669

$\overline{21}$ Nijenhuis ER. Somatoform Dissociation. Phenomena, measurement and the theoretical issues. Van Gorcum \& Comp Assen, The Netherlands: 1999

$\overline{22}$ Reddemann L. Psychodynamisch-imaginative Traumatherapie, PITT. Das Manual. 3. Aufl. Stuttgart: Klett-Cotta; 2008

$\overline{23}$ Reddemann L, Liebermann P, Söllner W et al. Stabilisierung. In: Seidler GH, Freyberger HJ, Maercker A, Hrsg. Handbuch der Psychotraumatologie. 2. Aufl. Stuttgart: Klett-Cotta; 2015: 639-655

$\overline{24}$ Schubert CS, Venkat S, Söllner W. Die Veränderung der gesundheitsbezogenen Lebensqualität bei chronischen Schmerzpatienten im Rahmen eines multimodalen Therapieprogramms - Ein Vergleich von Patienten mit der Diagnose „Anhaltende somatoforme Schmerzstörung " und anderen chronischen Schmerzpatienten (Abstract). Psychother Psych Med 2009; 59: 90-91

$\overline{25}$ Söllner W, Schüßler G. Psychodynamische Therapie bei chronischen Schmerzerkrankungen. Eine systematische Literaturübersicht. Z Psychosom Med Psychother 2001; 47: 115-139

$\overline{26}$ Söllner W, Venkat S. Starke Schmerzen in der Fremde: Patienten mit Migrationshintergrund. Münch Med Wschr 2012; 18: 76-78

$\overline{27}$ Streltzer J, Linden M. Erhöhte Schmerzempfindlichkeit unter Dauerbehandlung mit Opiaten. Nervenarzt 2008; 79 (5): 607-611

$\overline{28}$ Winnicott DW. Reifungsprozesse und fördernde Umwelt. München: Kindler; 1974

$\overline{29}$ Wöller W. Traumaspezifische Behandlungstechniken. Psychotherapie im Dialog 2006; 7 (4): 405-407

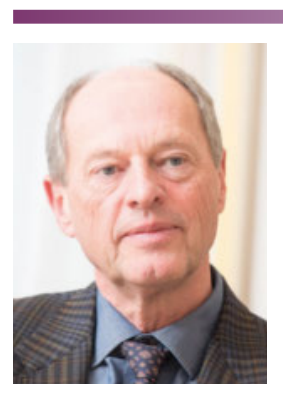

Univ.-Prof. Dr. med. univ. Wolfgang Söllner Universitätsklinik für Psychosomatische Medizin und Psychotherapie und Interdisziplinäre Schmerztagesklinik, Paracelsus Medizinische Privatuniversität

Klinikum Nürnberg

Prof. Ernst-Nathan-Str. 1

90419 Nürnberg

wolfgang.soellner@klinikum-nuernberg.de

Wolfgang Söllner ist seit 2002 Chefarzt der Klinik für Psychosomatische Medizin und Psychotherapie am Klinikum Nürnberg. Seit 2014 Vize-Rektor der Paracelsus Medizinischen Privatuniversität Nürnberg. Zu seinen Arbeitsschwerpunkten zählen u. a. Psychosomatik in der Schmerztherapie, Psychosomatische Aspekte und Psychotherapie mit körperlich Kranken sowie die Behandlung von Angststörungen, depressiven Störungen, Belastungsreaktionen, Identitäts- und Selbstwertstörungen.

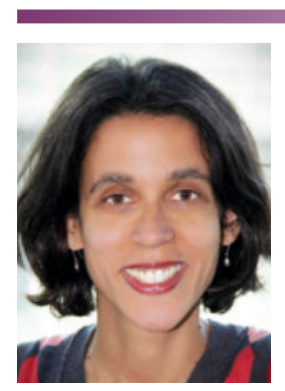

Lic. phil. Sandra Venkat

Interdisziplinäre Schmerztagesklinik, Paracelsus Medizinische Privatuniversität sandra.venkat@klinikum-nuernberg.de

Sandra Venkat ist klinische Psychologin und psychologische Psychotherapeutin (kognitive Verhaltenstherapie) sowie Supervisorin für Spezielle Schmerzpsychotherapie. Sie ist als leitende Psychologin der Schmerztagesklinik am Klinikum Nürnberg Nord tätig. Arbeitsschwerpunkt: therapeutische Arbeit mit Schmerzpatienten mit Migrationshintergrund. 